\title{
RESEARCH
}

Open Access

\section{Cost-effectiveness analysis of using the TBX6-associated congenital scoliosis risk score (TACScore) in genetic diagnosis of congenital scoliosis}

Zefu Chen ${ }^{1,2,3 \dagger}$, Zihui Yan ${ }^{1,2,3 \dagger}$, Chenxi Yu ${ }^{1,2,3 \dagger}$, Jiaqi Liu ${ }^{1,2,4}$, Yanbin Zhang ${ }^{1,2,3}$, Sen Zhao ${ }^{1,2}$, Jiachen Lin ${ }^{1,2,3}$,

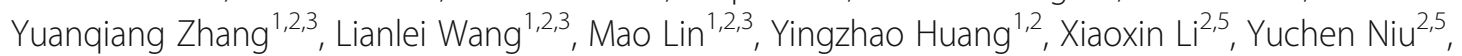
Shengru Wang ${ }^{1,2,6}$, Zhihong Wu ${ }^{2,5,6}$, DISCO (Deciphering disorders Involving Scoliosis and COmorbidities) study group, Guixing Qiu ${ }^{1,2,5,6}$, Terry Jianguo Zhang ${ }^{1,2,6}$ and Nan $\mathrm{Wu}^{1,2,6^{*}}$

\footnotetext{
Abstract

Background: We previously reported a novel clinically distinguishable subtype of congenital scoliosis (CS), namely, TBX6-associated congenital scoliosis (TACS). We further developed the TBX6-associated CS risk score (TACScore), a multivariate phenotype-based model to predict TACS according to the patient's clinical manifestations. In this study, we aimed to evaluate whether using the TACScore as a screening method prior to performing whole-exome sequencing (WES) is more cost-effective than using WES as the first-line genetic test for CS.

Methods: We retrospectively collected the molecular data of 416 CS patients in the Deciphering disorders Involving Scoliosis and COmorbidities (DISCO) study. A decision tree was constructed to estimate the cost and the diagnostic time required for the two alternative strategies (TACScore versus WES). Bootstrapping simulations and sensitivity analyses were performed to examine the distributions and robustness of the estimates. The economic evaluation considered both the health care payer and the personal budget perspectives.

\footnotetext{
* Correspondence: dr.wunan@pumch.cn

${ }^{\dagger}$ Zefu Chen, Zihui Yan and Chenxi Yu contributed equally to this work.

'Department of Orthopedic Surgery, Peking Union Medical College Hospital,

Peking Union Medical College and Chinese Academy of Medical Sciences, Beijing 100730, China

${ }^{2}$ Beijing Key Laboratory for Genetic Research of Skeletal Deformity, Beijing 100730, China

Full list of author information is available at the end of the article
}

C C The Author(s). 2020 Open Access This article is licensed under a Creative Commons Attribution 4.0 International License, which permits use, sharing, adaptation, distribution and reproduction in any medium or format, as long as you give appropriate credit to the original author(s) and the source, provide a link to the Creative Commons licence, and indicate if changes were made. The images or other third party material in this article are included in the article's Creative Commons licence, unless indicated otherwise in a credit line to the material. If material is not included in the article's Creative Commons licence and your intended use is not permitted by statutory regulation or exceeds the permitted use, you will need to obtain permission directly from the copyright holder. To view a copy of this licence, visit http://creativecommons.org/licenses/by/4.0/. The Creative Commons Public Domain Dedication waiver (http://creativecommons.org/publicdomain/zero/1.0/) applies to the data made available in this article, unless otherwise stated in a credit line to the data. 
(Continued from previous page)

Results: From the health care payer perspective, the strategy of using the TACScore as the primary screening method resulted in an average cost of $\$ 1074.2$ (95\%Cl: $\$ 1044.8$ to $\$ 1103.5)$ and an average diagnostic duration of 38.7d (95\%Cl: $37.8 \mathrm{~d}$ to $39.6 \mathrm{~d}$ ) to obtain a molecular diagnosis for each patient. In contrast, the corresponding values were $\$ 1169.6$ (95\%Cl: \$1166.9 to \$1172.2) and 41.4d (95\%Cl: 41.1d to 41.7d) taking WES as the first-line test $(P<0.001)$. From the personal budget perspective, patients who were predicted to be positive by the TACScore received a result with an average cost of $\$ 715.1$ (95\%Cl: $\$ 594.5$ to $\$ 835.7$ ) and an average diagnostic duration of 30.4d (95\%Cl: $26.3 \mathrm{~d}$ to 34.6d). Comparatively, the strategy of WES as the first-line test was estimated to have significantly longer diagnostic time with an average of $44.0 \mathrm{~d}$ (95\%Cl: $43.2 \mathrm{~d}$ to $44.9 \mathrm{~d})$, and more expensive with an average of \$1193.4 (95\%Cl: \$1185.5 to \$1201.3) $(P<0.001)$. In 100\% of the bootstrapping simulations, the TACScore strategy was significantly less costly and more time-saving than WES. The sensitivity analyses revealed that the TACScore strategy remained cost-effective even when the cost per WES decreased to $\$ 8.8$.

Conclusions: This retrospective study provides clinicians with economic evidence to integrate the TACScore into clinical practice. The TACScore can be considered a cost-effective tool when it serves as a screening test prior to performing WES.

Keywords: TBX6-associated congenital scoliosis, TBX6-associated congenital scoliosis risk score, Whole-exome sequencing, Molecular diagnosis, Cost-effectiveness analysis

\section{Background}

Congenital scoliosis (CS), a relatively rare disease with a prevalence of approximately $0.5-1$ per 1000 live births, is a kind of spinal curvature that results from vertebral malformations, including formation failure, segmentation failure, or a combination of the two $[1,2]$. Genetic factors, vitamin deficiency, chemicals and environmental factors are assumed to independently or jointly contribute to CS $[3,4]$.

To date, with multiple clinical genetic measurements, we are able to molecularly classify CS according to their genetic etiologies [5]. We recently reported a novel clinically distinguishable subtype of CS, namely, TBX6-associated congenital scoliosis (TACS). Briefly, TACS is caused by TBX6 loss-of-function variations compound with the common hypomorphic single nucleotide polymorphisms (defined by rs2289292-rs3809624-rs3809627), and is characterized by hemivertebrae or butterfly vertebrae in the lower half of the spine [6-9]. Compared with non-TACS patients, TACS patients demonstrate simpler rib anomalies and fewer intraspinal malformations. Based on the specific phenotype-genotype relationship, we developed the TBX6-associated CS risk score (TACScore), a multivariate phenotype-based model to predict TACS according to the patient's clinical manifestations [7]. Specifically, the TACScore integrates the following variables: 1) segmented hemivertebrae or segmented butterfly vertebrae involving the lower half of the spine (T8-S5) (0 or 3 points); 2$)$ the number of vertebral malformations ( -4 to 0 points); 3 ) the presence of intraspinal defects ( 3 or 0 points); and 4 ) the types of rib defect ( -1 to 1 points) (Table S1). The cutoff point of the TACScore was selected as 3 to achieve a high Youden index, with a sensitivity, specificity, and accuracy of $93.9 \%, 90.9 \%$ and $91.2 \%$ respectively [7].
Currently, whole-exome sequencing (WES) is recommended in clinical practice for patients suspected of Mendelian diseases, and is supposed to be implemented at the early stage of diagnosis to improve the costeffectiveness [10]. However, compared with single-gene or a subset of genes (i.e. panel) test, WES is sometimes still considered costly and time-consuming for patients [11]. In this study, we aimed to compare whether applying the TACScore as a screening test prior to performing WES is more cost-effective than WES as the firstline test in molecularly diagnosing CS.

\section{Results}

We retrospectively collected the molecular data of 416 patients from the Deciphering disorders Involving Scoliosis and COmorbidities (DISCO) study. We designed a decision tree (Fig.1) and performed a cost-effectiveness analysis for all participants. The cost-effectiveness analysis focused on the costs and diagnostic times along the diagnostic trajectory: outpatient appointment, DNA extraction and shipping, WES, Sanger sequencing, droplet digital polymerase chain reaction (ddPCR), genetic consultations and clinical consultations (Table 1).

\section{Cost-effectiveness analysis from the health care payer perspective}

In counterfactual model 1 (the WES strategy), WES diagnosed a total of 73/416 (17.5\%) patients, 42 of whom were diagnosed by TBX6 variations (thus TACS) and 31 of whom were diagnosed by other genes [12]. The total cost of 416 patients was $\$ 486,545.4$, and the total diagnostic time was $17,224 \mathrm{~d}$. The average cost per patient was \$1169.6 (95\% CI: \$1166.9 to $\$ 1172.2$ ). The average 


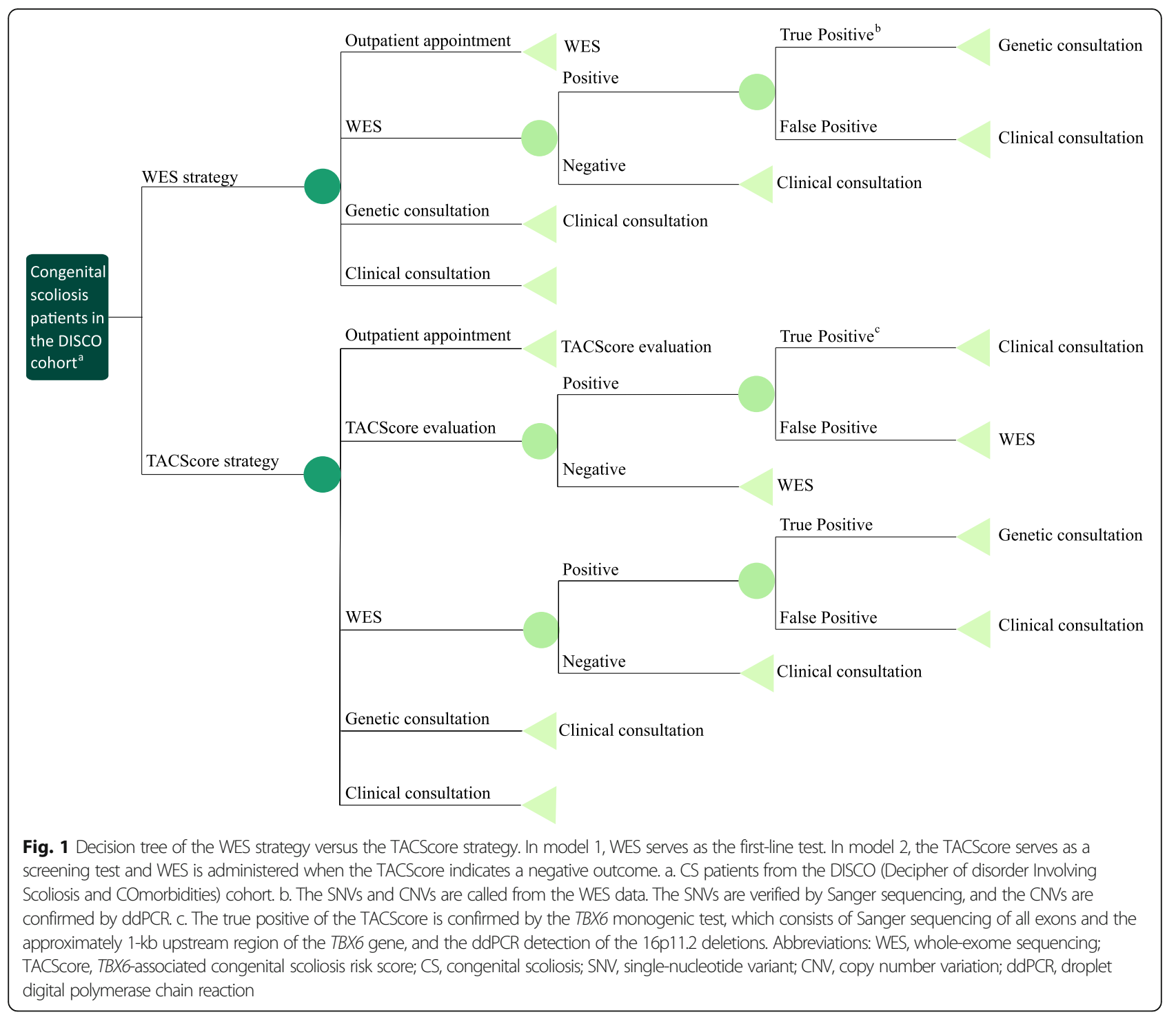

diagnostic time per patient was $41.4 \mathrm{~d}$ (95\% CI: $41.1 \mathrm{~d}$ to $41.7 \mathrm{~d})$.

In counterfactual model 2 (the TACScore strategy), $83 / 416(20.0 \%)$ patients were predicted to be positive by

Table 1 The costs and diagnostic times required for investigations

\begin{tabular}{lll}
\hline Variables & Cost (USD) & Time (d) \\
\hline Outpatient appointment & 16.1 & 1 \\
DNA extraction and shipping & 29.9 & 1 \\
Whole-exome sequencing & 1094.8 & 37 \\
Sanger sequencing & 23.7 & 7 \\
ddPCR & 32.2 & 7 \\
Genetic consultation & 16.1 & 1 \\
Clinical consultation & 16.1 & 1 \\
\hline
\end{tabular}

Abbreviations: $d d P C R$ droplet digital polymerase chain reaction the TACScore (TACScore $\geq 3$ ) and 42 were diagnosed with TACS by the TBX6 monogenic test (including Sanger sequencing and ddPCR). Subsequently, a total of 374 patients underwent WES, including 31 patients who were further diagnosed by other genes and 343 patients who received uninformative WES reports. The average cost per patient was reduced to $\$ 1074.2$ (95\% CI: $\$ 1044.8$ to $\$ 1103.5)$, and the average diagnostic time per patient decreased to $38.7 \mathrm{~d}$ (95\% CI: $37.8 \mathrm{~d}$ to $39.6 \mathrm{~d}$ ).

Compared with using WES as the first-line test (counterfactual model 1), using the TACScore as a primary screening prior to performing WES (counterfactual model 2) saved $\$ 95.4$ (95\% CI: $\$ 66.0$ to $\$ 124.9, P<$ $0.001)$ and $2.7 \mathrm{~d}(95 \% \mathrm{CI}: 1.7 \mathrm{~d}$ to $3.7 \mathrm{~d}, \quad \mathrm{P}<0.001)$ (Table 2). The TACScore strategy and the WES strategy shared an identical diagnostic rate $(73 / 416,17.5 \%)$. In this study, the incremental cost-effectiveness ratio 
Table 2 Cost-effectiveness of the TACScore strategy versus the WES strategy from the health care payer perspective

\begin{tabular}{|c|c|c|}
\hline & $\begin{array}{l}\text { Model 1: } \\
\text { WES as a first- } \\
\text { line test (USD) }\end{array}$ & $\begin{array}{l}\text { Model 2: } \\
\text { TACScore as a } \\
\text { screening test (USD) }\end{array}$ \\
\hline \multicolumn{3}{|l|}{ Assessments } \\
\hline Outpatient appointment & 6697.6 & 6697.6 \\
\hline Genetic consultation & 1175.3 & 563.5 \\
\hline Clinical consultation & 6697.6 & 6697.6 \\
\hline \multicolumn{3}{|l|}{ TBX6 Monogenic test } \\
\hline Sanger sequencing & 1730.1 & 2796.6 \\
\hline$d d P C R$ & 2350.6 & 3799.6 \\
\hline \multicolumn{3}{|l|}{ Next generation sequencing } \\
\hline WES & 455436.8 & 413834.4 \\
\hline \multicolumn{3}{|l|}{ Other } \\
\hline $\begin{array}{l}\text { DNA extraction and } \\
\text { shipping }\end{array}$ & 12457.5 & 12457.5 \\
\hline Total cost & 486545.4 & 446846.8 \\
\hline Total diagnostic time $(\mathrm{d})$ & 17224 & 16095 \\
\hline Total number of patients & 416 & 416 \\
\hline $\begin{array}{l}\text { Number of patients } \\
\text { underwent WES }\end{array}$ & 416 & 378 \\
\hline Total number of diagnoses & 73 & 73 \\
\hline $\begin{array}{l}\text { Average cost per patient } \\
(95 \% \mathrm{Cl})\end{array}$ & $\begin{array}{l}1169.6(1166.9 \\
1172.2)\end{array}$ & $1074.2(1044.8,1103.5)$ \\
\hline $\begin{array}{l}\text { Average cost difference } \\
(95 \% \mathrm{Cl})\end{array}$ & - & $-95.4(-124.9,-66.0)$ \\
\hline $\begin{array}{l}\text { Average diagnostic time per } \\
\text { patient }(95 \% \mathrm{Cl})(\mathrm{d})\end{array}$ & $41.4(41.1,41.7)$ & $38.7(37.8,39.6)$ \\
\hline $\begin{array}{l}\text { Average diagnostic time } \\
\text { difference }(95 \% \mathrm{Cl})(\mathrm{d})\end{array}$ & - & $-2.7(-3.7,-1.7)$ \\
\hline
\end{tabular}

Abbreviations: WES whole-exome sequencing, TACScore TBX6-associated congenital scoliosis risk score, $d d P C R$ droplet digital polymerase chain reaction

(ICER) was not reported because the TACScore strategy is not only less costly but also more time-saving than the WES strategy.

\section{Cost-effectiveness from the personal budget perspective}

Regarding the patients who were predicted to be positive by the TACScore, in counterfactual model 1 (the WES strategy), WES diagnosed a total of $42 / 83$ (17.5\%) patients, 38 of whom were diagnosed by TBX6 variations (thus TACS) and 4 of whom were diagnosed by other genes. The total cost of 83 patients was 99,050.5\$, and the total diagnostic time was 3656d. The average cost per patient was $\$ 1193.4$ (95\% CI: $\$ 1185.5$ to $\$ 1201.3$ ). The average diagnostic time per patient was $44.0 \mathrm{~d}(95 \%$ CI: $43.1 \mathrm{~d}$ to $44.9 \mathrm{~d})$.

In counterfactual model 2 (the TACScore strategy), 38 were diagnosed with TACS by the TBX6 monogenic test (including Sanger sequencing and ddPCR). Subsequently, a total of 45 patients underwent WES, including 4 patients who were further diagnosed by other genes and 41 patients who received uninformative WES reports. The total cost of 83 patients was 59,351.8\$, and the total diagnostic time was $2527 \mathrm{~d}$. The average cost per patient was $\$ 715.1$ (95\% CI: $\$ 594.5$ to $\$ 835.7$ ) and the average diagnostic time per patient was $30.4 \mathrm{~d}$ (95\% CI: $26.3 \mathrm{~d}$ to $34.6 \mathrm{~d})$.

Compared with using WES as the first-line test (counterfactual model 1), using the TACScore as a primary screening prior to performing WES (counterfactual model 2) saved $\$ 478.3$ (95\% CI: $\$ 357.5$ to $\$ 599.1, P<$ 0.001 ) and $13.6 \mathrm{~d}$ (95\% CI: $9.4 \mathrm{~d}$ to $17.8 \mathrm{~d}, \mathrm{P}<0.001)$ (Table 3).

\section{Bootstrapped simulations}

To confirm our findings in a larger population and estimate the reliability of the calculations, we created 500

Table 3 Cost-effectiveness of the TACScore strategy versus the WES strategy from the personal budget perspective

\begin{tabular}{|c|c|c|}
\hline & $\begin{array}{l}\text { Model 1: } \\
\text { WES as a first- } \\
\text { line test (USD) }\end{array}$ & $\begin{array}{l}\text { Model 2: } \\
\text { TACScore as a } \\
\text { screening test (USD) }\end{array}$ \\
\hline \multicolumn{3}{|l|}{ Assessments } \\
\hline Outpatient appointment & 1336.3 & 1336.3 \\
\hline Genetic consultation & 676.2 & 64.4 \\
\hline Clinical consultation & 1336.3 & 1336.3 \\
\hline \multicolumn{3}{|l|}{ TBX6 Monogenic test } \\
\hline Sanger sequencing & 995.4 & 2061.9 \\
\hline $\mathrm{ddPCR}$ & 1352.4 & 2801.4 \\
\hline \multicolumn{3}{|l|}{ Next generation sequencing } \\
\hline WES & 90868.4 & 49266.0 \\
\hline \multicolumn{3}{|l|}{ Other } \\
\hline $\begin{array}{l}\text { DNA extraction and } \\
\text { shipping }\end{array}$ & 2485.5 & 2485.5 \\
\hline Total cost & 99050.5 & 59351.8 \\
\hline Total diagnostic time $(\mathrm{d})$ & 3656 & 2527 \\
\hline Total number of patients & 83 & 83 \\
\hline $\begin{array}{l}\text { Number of patients } \\
\text { underwent WES }\end{array}$ & 83 & 45 \\
\hline Total number of diagnoses & 42 & 42 \\
\hline $\begin{array}{l}\text { Average cost per patient } \\
(95 \% \mathrm{Cl})\end{array}$ & $\begin{array}{l}1193.4(1185.5 \\
1201.3)\end{array}$ & $715.1(594.5,835.7)$ \\
\hline $\begin{array}{l}\text { Average cost difference } \\
(95 \% \mathrm{Cl})\end{array}$ & - & $\begin{array}{l}-478.3(-599.1 \\
-357.5)\end{array}$ \\
\hline $\begin{array}{l}\text { Average diagnostic time per } \\
\text { patient }(95 \% \mathrm{Cl})(\mathrm{d})\end{array}$ & $44.0(43.2,44.9)$ & $30.4(26.3,34.6)$ \\
\hline $\begin{array}{l}\text { Average diagnostic time } \\
\text { difference }(95 \% \mathrm{Cl})(\mathrm{d})\end{array}$ & - & $-13.6(-17.8,-9.4)$ \\
\hline
\end{tabular}

Abbreviations: WES whole-exome sequencing, TACScore TBX6-associated congenital scoliosis risk score, $d d P C R$ droplet digital polymerase chain reaction 
datasets. Each dataset consisted of 500 random samples from the study participants' records. The cost and diagnostic time were calculated for each sample, and subsequently the average cost per patient and average diagnostic time per patient were calculated for each dataset. Thus, the average cost and diagnostic time difference were determined in each dataset. The costeffectiveness plane demonstrated that the TACScore as a primary screening method was less costly and more time-saving than WES as the first-line test in $100 \%$ of the 500 simulations (Fig.2).

\section{Sensitivity analyses}

To estimate the robustness of the cost-effectiveness analysis, we performed the one-way sensitivity analysis to examine how each variable affected the cost and diagnostic time. In terms of cost, the most influential variables were the costs of WES, Sanger sequencing, ddPCR and genetic consultation. The TACScore strategy remained cost-effective unless the cost of WES decreased to $\$ 8.8$, or the costs of Sanger sequencing or ddPCR increased to $\$ 562.5$ or $\$ 571.1$, respectively. Regarding the diagnostic time, the TACScore strategy remained time-saving unless WES required less than $13 \mathrm{~d}$, or Sanger sequencing or ddPCR required more than 20d (Table 4).

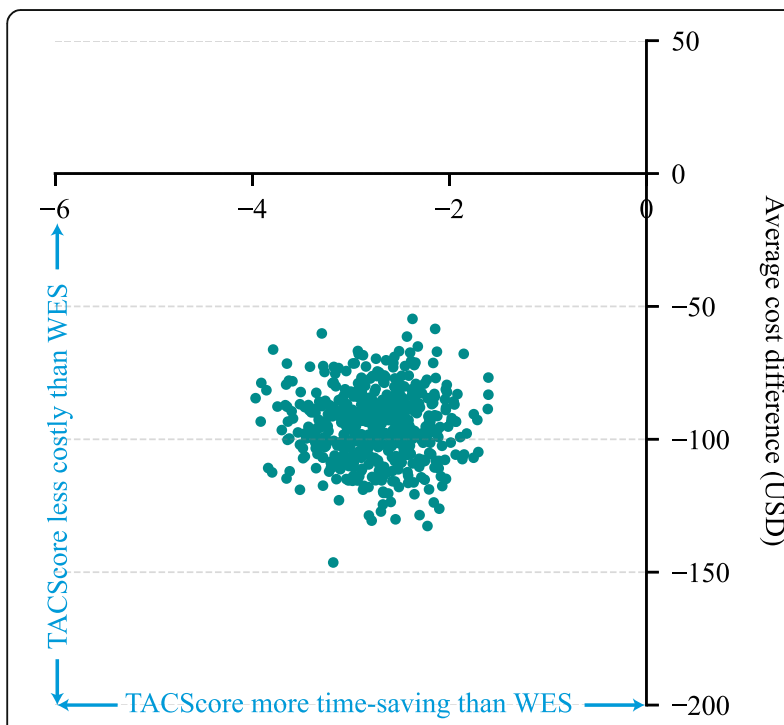

Average diagnostic time difference (d)

Fig. 2 Cost-effectiveness plane for the WES strategy versus the TACScore strategy. The scatter plot contains 500 points, and each point represents the average cost differences and average diagnostic time differences of 500 bootstrapped simulations. Points below the $x$-axis and on the left side of the $y$-axis indicate that the TACScore strategy is less costly and more time-saving than WES. Therefore, for all of these simulations, the TACScore is considered cost-effective. Abbreviations: WES, whole-exome sequencing; TACScore, TBX6-associated congenital scoliosis risk score
The two-way sensitivity analysis of the prevalence of TACS in CS versus the price of WES revealed that the preferred strategy varied (Fig.3a). Specifically, above the red line, the TACScore strategy was more cost-effective than the WES strategy. The two-way sensitivity analysis of the 1-specificity of the TACScore versus the sensitivity of the TACScore was demonstrated in Fig.3b. In detail, above the red line, the TACScore strategy remained less costly than the WES strategy. Above the blue line, the TACScore strategy remained more time-saving.

\section{Discussion}

The TACScore provides clinical geneticists with an efficient tool to evaluate the likelihood of a CS patient being diagnosed with TACS and, whether the patient is recommended to undergo a single gene or a genome-scale genetic test. Using the TACScore as the primary screening method counterfactually in the DISCO cohort, which provided a real and reliable molecular diagnostic yield of CS, improved the cost-effectiveness of the diagnostic process. Our study provides robust economic evidence for the translation from using the TACScore as a clinical assessment tool toward its application as a cost-effective screening test in molecularly diagnosing CS patients.

Delayed diagnosis and misdiagnosis are common for rare diseases and may cause serious and adverse effects on the patients [13-17]. Specifically, delayed diagnosis and misdiagnosis in the TACS lead to emotional distress, progression of scoliosis and delayed treatment. Besides, families whose child has a delayed diagnosis often consult multiple clinicians prior to receiving the correct molecular diagnosis [17]. An ill-defined genetic risk, frustration and disease progression brought by the diagnostic odyssey can cause anxiety and loss of reproductive confidence. In this condition, the patients' families need to access the health system for treatment of anxiety and depression and pay additional medical costs. Thus, a prompt and accurate diagnosis can prevent disease progression, improve quality of life, and reduce financial burden. According to a recent study, lack of knowledge among health professionals, delays in obtaining test results and lack of access to appropriate tests are the leading causes of diagnostic delays [17]. Therefore, an accessible, fast and accurate screening test is needed for diagnosing TACS, which could be enabled by the TACScore. Evaluating patients' phenotypes by the TACScore is both economical and time-saving, while WES is expensive, time-consuming and sometimes hard to access. The TACScore strategy successfully lowers the cost of diagnostic tests, and consequently more CS patients can receive a molecular diagnosis in time. Furthermore, a fast and accurate diagnosis provided by the TACScore can help the patients' families alleviate the stress of disease progression, and enable them to end the diagnostic 
Table 4 One-way sensitivity analysis for each variable in the cost-effectiveness analysis

\begin{tabular}{lll}
\hline Variables & Baseline Value & Range \\
\hline Cost (USD) & 16.1 & \\
Outpatient appointment & 29.9 & Always \\
DNA extraction and shipping & 1094.8 & Always \\
WES & 23.7 & $<8.8$ \\
Sanger sequencing & 32.2 & $>562.5$ \\
ddPCR & 16.1 & $>571.1$ \\
Genetic consultation & 16.1 & Always \\
Clinical consultation & & Always \\
Time (d) & 1 & \\
Outpatient appointment & 1 & Always \\
DNA extraction and shipping & 37 & Always \\
WES & 7 & $<13$ \\
Sanger sequencing & 7 & $>20$ \\
ddPCR & 1 & $>20$ \\
Genetic consultation & 1 & Always \\
Clinical consultation & Always \\
\hline
\end{tabular}

Abbreviations: WES whole-exome sequencing, $d d P C R$ droplet digital polymerase chain reaction

odyssey, and to restore reproductive confidence. In this case, using the TACScore as the first-line screening test has the capacity to improve CS patients' prognosis by offering a prompt and correct molecular diagnosis.

A recent study found that a rare diseases cohort representing approximately $2.0 \%$ of an Australian state population accounted for between 4.6 and $10.5 \%$ of the state inpatient hospital costs, highlighting the marked disparity between the rare diseases population and their combined health-system costs $[18,19]$. Interpretation of this result must take into consideration that a lack of knowledge and experience among health professionals lead to delayed and inaccurate diagnosis, followed by inappropriate referral pathways and expensive diagnostic procedures [10, 14, 19-22]. Specifically, CS impairs physical and mental abilities, shortens life expectancy and places a significant economic burden on the healthcare system [23]. With the inclusion of outpatient, mental health, and rehabilitation expenses, the total healthsystem cost attributed to CS is substantially greater than expected. Thus, to alleviate the significant financial burden of CS, we need an improved access to a costeffective diagnostic test. As the TACScore is a simple and practical phenotype-based model, the health professionals can understand the characteristics of TACS readily and recognize its specific clinical phenotypes easily. Therefore, the health professionals can better identify TACS, order diagnostic tests and make an early diagnosis. Besides, as demonstrated by this study, using the TACScore as a screening test is less expensive than performing WES as the first-line test. Taken together, implementing the TACScore as a screening test can cut down the cost of CS patients to a level that is more proportionate to the prevalence of this rare condition.

This study also has some limitations. Although economic information, which supports the general use of
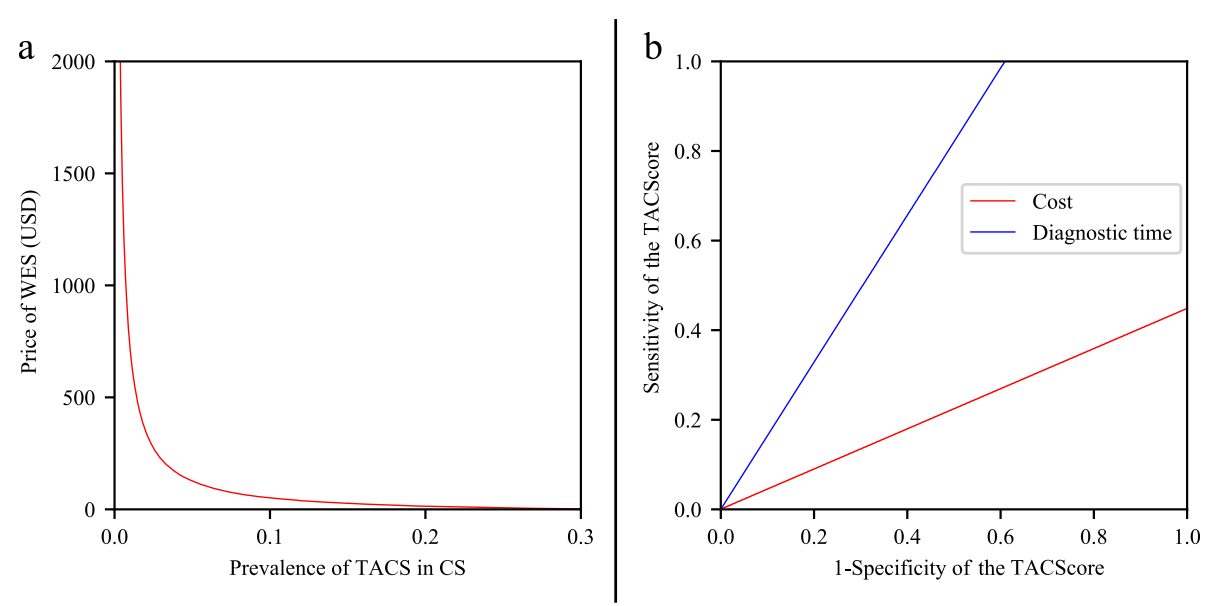

Fig. 3 Two-way sensitivity analysis. a Two-way sensitivity analysis of the prevalence rate of TACS in CS versus the cost of WES. The $x$-axis shows the hypothesized prevalence of TACS in CS. The $y$-axis shows the threshold of the WES price. Above the red line, the TACScore is more costeffective than the WES strategy. Under the red line, the WES strategy is more cost-effective than the TACScore strategy. $\mathbf{b}$ Two-way sensitivity analysis of the sensitivity of the TACScore versus the 1-specificity of the TACScore. The x-axis shows the hypothesized 1-specificity of the TACScore. The $y$-axis shows the hypothesized sensitivity of the TACScore. Above the red line, the TACScore strategy is less costly than the WES strategy. Above the blue line, the TACScore strategy is more time-saving than the WES strategy. Abbreviations: TACS, TBX6-associated congenital scoliosis; CS, congenital scoliosis; WES, whole-exome sequencing; TACScore, TBX6-associated congenital scoliosis risk score 
the TACScore as screening test in molecularly diagnosing CS, was provided by this study, individual hospitals still need to evaluate the feasibility of using the TACScore in clinical practice. In China, WES is not in the scope of government medical insurance. Therefore, we conducted a cost-effectiveness analysis without considering different reimbursement rates. We admit that while the decision tree of the cost-effectiveness analysis varies from country to country, system to system, the TACScore system is still needed to be validated in other healthcare systems. This study collected the CS patients retrospectively, and designed the two models counterfactually, which merited further prospective studies to obtain high-level evidence.

\section{Conclusions}

In summary, this retrospective study provides clinicians with economic evidence to integrate the TACScore into clinical practice. The TACScore can be considered a cost-effective tool when it serves as a screening test prior to performing WES.

\section{Methods}

\section{Study design}

This is a retrospective study to determine whether using the TACScore as a screening test for CS patients is more cost-effective than WES as the first-line test. We designed a cost-effectiveness analysis decision tree (Fig.1) using previously described techniques [24-27]. The diagnosis-related costs were analyzed to determine the differences of average cost and diagnostic duration between the two strategies.

\section{Patients collection}

The DISCO cohort, which consisted of unrelated sporadic CS patients of Chinese Han descent, was recruited from Peking Union Medical College Hospital (PUMCH) between October 2010 and January 2018 [7]. Patients in DISCO cohort were retrospectively identified for possible inclusion in this study. The inclusion criteria were as follows: 1) patients who were diagnosed with CS; 2) patients who had available data regarding onset age, age of enrollment, sex and complete spinal images including $\mathrm{X}$-ray, computed tomography $(\mathrm{CT})$, and magnetic resonance imaging (MRI); 3) patients underwent WES for pursuing a molecular diagnosis; and 4) patients with informed consent for enrollment. The study was approved by the institutional review board of PUMCH.

\section{Cost-effectiveness analysis}

We compared the differences of the cost and diagnostic duration under two counterfactual strategies (Fig.1):
The WES strategy, WES as the first-line test (counterfactual model 1). We modeled this counterfactual diagnostic pathway in which all recruited patients received WES as the first-line test.

The TACScore strategy, the TACScore as a screening test (counterfactual model 2). Patients were evaluated by the TACScore first. A TBX6 monogenic test was performed for patients who had TACScore was $\geq 3$. WES was implemented when the TACScore was $\leq 2$.

For both of the strategies, the price of DNA extraction and shipping were calculated once, and each instance of a positive WES result required an appointment with a geneticist to interpret the report.

From the health care payer perspective, the overall expenditure and diagnostic time for all enrolled patients were calculated according to two diagnostic counterfactual strategies. From the personal budget perspective, the personal expenditure and diagnostic time for patients with a positive TACScore were calculated according to two diagnostic counterfactual strategies.

\section{WES and TBX6 monogenic test}

WES was performed in all patients as described previously $[6,28]$. Sanger sequencing or/and ddPCR were performed to validate the causative variants.

TBX6 monogenic test included Sanger sequencing of all exons and the approximately $1-\mathrm{kb}$ upstream region of the TBX6 gene and ddPCR testing of the $16 \mathrm{p} 11.2$ deletions [6]. For patients who underwent both Sanger sequencing and ddPCR, the one which was more timeconsuming was regarded as the diagnostic time.

\section{Costs and diagnostic times}

We obtained the costs and diagnostic times of the whole process of molecularly diagnosing a patient, which included outpatient appointment, DNA extraction and shipping, WES, Sanger sequencing, ddPCR, genetic consultation and clinical consultation (Table 1). The aforementioned data complied with the Chinese Medical Service Price Regulations. The total unit cost and diagnostic time of each patient were derived according to the decision tree (Fig.1). We converted Chinese Yuan (CNY) into American Dollars (USD) based on the exchange rate of CNY $1=$ USD 0.161 on 30 June 2014 (source: http://fx.cmbchina.com). The duration of the diagnostic trajectory was calculated from the first clinical visit to when a genetic report was issued.

\section{Bootstrapped simulations and sensitivity analyses}

We performed 500 bootstraps to estimate the reliability of the analysis. Each bootstrap represented 500 samples randomly collected from our cohort. We calculated the outcomes (including the average cost and the diagnostic 
time per patient) for each replicated data set, and then evaluated the distribution of each outcome.

We performed one-way and two-way sensitivity analyses to examine the robustness of the estimates. For the one-way sensitivity analysis, we determined the thresholds of the variables that alter the most cost-effective strategy according to the decision tree. For the two-way sensitivity analysis, we changed two variables simultaneously. Specifically, the two-way analysis was performed for two pairs of influential values (i.e. the prevalence of TACS in CS versus the price of WES, and 1-specificity of the TACScore versus sensitivity of the TACScore).

\section{Statistical analysis}

We used the Student's t-test for analyses of continuous variables. Statistical significance for differences was set as $P=0.05$ (2-sided). The statistical analyses were performed using Python (version 3.7) and SPSS (version 21.0).

\section{Supplementary information}

Supplementary information accompanies this paper at https://doi.org/10. 1186/s13023-020-01537-y.

\section{Additional file 1.}

\section{Abbreviations}

CS: congenital scoliosis; TACS: TBX6-associated congenital scoliosis; TACScore: TBX6-associated congenital scoliosis risk score; WES: whole-exome sequencing; DISCO: Deciphering disorders Involving Scoliosis and COmorbidities; ddPCR: droplet digital polymerase chain reaction; PUMCH: Peking Union Medical College Hospital

\section{Acknowledgments}

We would like to thank all the individuals involved in the study for their participation. We thank the nurses and doctors from the Department of Orthopedic Surgery of Peking Union Medical College Hospital for assistance with patients enrollment. We thank Nanjing Geneseeq Inc. for the techinical help in exome sequencing.

The complete author list of the DISCO study:

Guixing Qiu, M.D. ${ }^{1,2,3}$, Zhihong Wu, M.D., Ph.D. ${ }^{1,2,3}$, Jianguo Zhang, M.D. ${ }^{1,2}$, Nan Wu, M.D. ${ }^{1,2,3,5}$, Lan Zhu, M.D. ${ }^{6}$, Shengru Wang, M.D. ${ }^{1,2}$, Na Chen, M.D. ${ }^{6}$, Jiaqi Liu, M.D. ${ }^{2,7}$, Sen Liu, M.D. ${ }^{1,2}$, Yuzhi Zuo, M.D. ${ }^{1,2}$, Gang Liu, M.D. ${ }^{1,2}$, Yuangiang Zhang, M.D. ${ }^{1,2}$, Chenxi Yu, M.D. ${ }^{1,2}$, Sen Zhao, B.S. ${ }^{1,2}$, Lianlei Wang M.D. ${ }^{1,2}$, Yanxue Zhao, M.D. ${ }^{1,2}$, Weisheng Chen, M.D. ${ }^{1,2}$, Zihui Yan, Ph.D. ${ }^{1,2}$ Xinzhuang Yang, Ph.D. ${ }^{1,2}$, Hengqiang Zhao, M.S. ${ }^{1,2}$, Yuchen Niu, M.S.', Jingdan Chen, M.S. ${ }^{2}$, Xiaoxin Li, M.S. ${ }^{2}$, Huizi Wang, B.S. ${ }^{2}$, Zhi Zhao, B.S. ${ }^{2}$, Yiran Cui, B.S. ${ }^{2}$, Zixin Zhang, B.S. ${ }^{2}$, Zefu Chen M.D. ${ }^{1,2}$, Bowen Liu, B.S. ${ }^{1,2}, X_{i}$ Cheng, B.S. ${ }^{1,2}$, Mao Lin, M.D. ${ }^{1,2}$, Jiachen Lin, M.D. ${ }^{1,2}$, Huakang Du, B.S. ${ }^{1,2}$, Yaqi Li, B.S. ${ }^{1,2}$, Yi You, M.S. ${ }^{1,2}$

1. Department of Orthopedic Surgery, Peking Union Medical College Hospital, Peking Union Medical College and Chinese Academy of Medical Sciences, Beijing 100730, China.

2. Beijing Key Laboratory for Genetic Research of Skeletal Deformity, Beijing 100730, China.

3. Medical Research Center of Orthopedics, Chinese Academy of Medical Sciences, Beijing 100730, China.

4. Medical Research Center, Peking Union Medical College Hospital, Peking Union Medical College and Chinese Academy of Medical Sciences, Beijing, China.

5. Department of Molecular and Human Genetics, Baylor College of Medicine; Houston, TX 77030, USA.

6. Department of Obstetrics and Gynaecology, Peking Union Medical College Hospital, Peking Union Medical College and Chinese Academy of Medical Sciences, Beijing 100730, China.
7. Department of Breast Surgical Oncology, National Cancer Center/Cancer Hospital, Chinese Academy of Medical Sciences and Peking Union Medical College, Beijing 100021, China

\section{Authors' contributions}

NW directed the project. NW, JZ and GQ designed the study. ZC, ZY and CY were involved in data management and statistical analysis and wrote the first and subsequent drafts of the manuscript. ZC, ZY, CY, JQL, YBZ, SZ, JCL, $Y Q Z, L W, M L, Y H, X L, Y N$, and SW gathered detailed clinical information for the study. ZC, ZY, CY and SZ analyzed the data. SW, ZW, GQ, JZ and NW supervised the data analysis, and reviewed and commented on all drafts. All other authors coordinated the study and critically revised the manuscript. The author(s) read and approved the final manuscript.

\section{Funding}

This research was funded in part by the National Natural Science Foundation of China $(81822030$ to N.W., 81930068 and 81772299 to Z.W., 81772301 and 81972132 to G.Q., 81672123 and 81972037 to J.Z.), Beijing Natural Science Foundation (7191007 to Z.W.).), CAMS Initiative Fund for Medical Sciences (2016-I2M-3-003 to G.Q. and N.W., 2016-I2M-2-006 and 2017-I2M-2-001 to Z.W.), the National Key Research and Development Program of China (No. 2018YFC0910506 to N.W. and Z.W.)

\section{Availability of data and materials}

The datasets used and/or analyzed during the current study are available from the corresponding author on reasonable request.

Ethics approval and consent to participate

All participants gave informed consent. The study was approved by the institutional review board of Peking Union Medical College Hospital.

\section{Consent for publication}

Consent for publication was obtained from all participants.

\section{Competing interests}

The authors declare that they have no competing interests.

\section{Author details}

'Department of Orthopedic Surgery, Peking Union Medical College Hospital, Peking Union Medical College and Chinese Academy of Medical Sciences, Beijing 100730, China. ${ }^{2}$ Beijing Key Laboratory for Genetic Research of Skeletal Deformity, Beijing 100730, China. ${ }^{3}$ Graduate School of Peking Union Medical College, Beijing 100005, China. ${ }^{4}$ Department of Breast Surgical Oncology, National Cancer Center/Cancer Hospital, Chinese Academy of Medical Sciences and Peking Union Medical College, Beijing, China. ${ }^{5}$ Medical Research Center, Peking Union Medical College Hospital, Peking Union Medical College and Chinese Academy of Medical Sciences, Beijing, China. ${ }^{6}$ Key Laboratory of Big Data for Spinal Deformities, Chinese Academy of Medical Sciences, Beijing 100730, China.

Received: 23 September 2019 Accepted: 7 September 2020 Published online: 15 September 2020

\section{References}

1. Hedequist D, Emans J. Congenital scoliosis. J Am Acad Orthop Surg. 2004; 12(4):266-75.

2. Wynne-Davies R. Congenital vertebral anomalies: aetiology and relationship to spina bifida cystica. J Med Genet. 1975;12(3):280-8.

3. Hensinger RN. Congenital scoliosis: etiology and associations. Spine (Phila Pa 1976). 2009;34(17):1745-50.

4. Chen Y, Liu Z, Chen J, Zuo Y, Liu S, Chen W, et al. The genetic landscape and clinical implications of vertebral anomalies in VACTERL association. J Med Genet. 2016:53(7):431-7.

5. Yang Y, Muzny DM, Reid JG, Bainbridge MN, Willis A, Ward PA, et al. Clinical whole-exome sequencing for the diagnosis of mendelian disorders. $N$ Engl J Med. 2013;369(16):1502-11.

6. Wu N, Ming X, Xiao J, Wu Z, Chen X, Shinawi M, et al. TBX6 null variants and a common hypomorphic allele in congenital scoliosis. N Engl J Med. 2015;372(4):341-50.

7. Liu J, Wu N, Yang N, Takeda K, Chen W, Li W, et al. TBX6-associated congenital scoliosis (TACS) as a clinically distinguishable subtype of 
congenital scoliosis: further evidence supporting the compound inheritance and TBX6 gene dosage model. Genet Med. 2019;21(7):1548-58.

8. Yang N, Wu N, Zhang L, Zhao Y, Liu J, Liang X, et al. TBX6 compound inheritance leads to congenital vertebral malformations in humans and mice. Hum Mol Genet. 2019;28(4):539-47.

9. Chen W, Lin J, Wang L, Li X, Zhao S, Liu J, et al. TBX6 missense variants expand the mutational spectrum in a non-Mendelian inheritance disease. Hum Mutat. 2020;41(1):182-95.

10. Stark Z, Schofield D, Alam K, Wilson W, Mupfeki N, Macciocca I, et al. Prospective comparison of the cost-effectiveness of clinical whole-exome sequencing with that of usual care overwhelmingly supports early use and reimbursement. Genet Med. 2017;19(8):867-74.

11. Schwarze K, Buchanan J, Taylor JC, Wordsworth S. Are whole-exome and whole-genome sequencing approaches cost-effective? A systematic review of the literature. Genet Med. 2018:20(10):1122-30.

12. Zhao S, Zhang Y, Chen W, Li W, Wang S, Wang L, et al. Diagnostic yield and clinical impact of exome sequencing in early-onset scoliosis (EOS). J Med Genet. 2020;jmedgenet-2019-106823.

13. Zurynski Y, Frith K, Leonard H, Elliott E. Rare childhood diseases: how should we respond? Arch Dis Child. 2008;93(12):1071-4

14. Knight AW, Senior TP. The common problem of rare disease in general practice. Med J Aust. 2006;185(2):82-3.

15. Baynam G, Pachter N, McKenzie F, Townshend S, Slee J, Kiraly-Borri C, et al. The rare and undiagnosed diseases diagnostic service - application of massively parallel sequencing in a state-wide clinical service. Orphanet J Rare Dis. 2016:11(1):77.

16. Gahl WA, Mulvihill JJ, Toro C, Markello TC, Wise AL, Ramoni RB, et al. The $\mathrm{NIH}$ undiagnosed diseases program and network: applications to modern medicine. Mol Genet Metab. 2016;117(4):393-400.

17. Zurynski Y, Deverell M, Dalkeith T, Johnson S, Christodoulou J, Leonard H, et al. Australian children living with rare diseases: experiences of diagnosis and perceived consequences of diagnostic delays. Orphanet J Rare Dis. 2017:12(1):68.

18. Graf von der Schulenburg JM, Frank M. Rare is frequent and frequent is costly: rare diseases as a challenge for health care systems. Eur J Health Econ. 2015;16(2):113-8.

19. Walker CE, Mahede T, Davis G, Miller LJ, Girschik J, Brameld K, et al. The collective impact of rare diseases in Western Australia: an estimate using a population-based cohort. Genet Med. 2017:19(5):546-52.

20. Gatta G, van der Zwan JM, Casali PG, Siesling S, Dei Tos AP, Kunkler I, et al. Rare cancers are not so rare: the rare cancer burden in Europe. Eur J Cancer. 2011:47(17):2493-511.

21. Jaffe A, Zurynski Y, Beville L, Elliott E. Call for a national plan for rare diseases. J Paediatr Child Health. 2010:46(1-2):2-4.

22. Garcia M, Downs J, Russell A, Wang W. Impact of biobanks on research outcomes in rare diseases: a systematic review. Orphanet J Rare Dis. 2018; 13(1):202.

23. Hedequist D, Emans J. Congenital scoliosis: a review and update. J Pediatr Orthop. 2007;27(1):106-16.

24. Detsky AS, Naglie G, Krahn MD, Naimark D, Redelmeier DA. Primer on medical decision analysis: part 1--getting started. Med Decis Mak. 1997;17(2): $123-5$.

25. Detsky AS, Naglie G, Krahn MD, Redelmeier DA, Naimark D. Primer on medical decision analysis: part 2--building a tree. Med Decis Mak. 1997;17(2): 126-35.

26. Naglie G, Krahn MD, Naimark D, Redelmeier DA, Detsky AS. Primer on medical decision analysis: part 3--estimating probabilities and utilities. Med Decis Mak. 1997;17(2):136-41.

27. Krahn MD, Naglie G, Naimark D, Redelmeier DA, Detsky AS. Primer on medical decision analysis: part 4--analyzing the model and interpreting the results. Med Decis Mak. 1997;17(2):142-51.

28. Wang K, Zhao S, Liu B, Zhang Q, Li Y, Liu J, et al. Perturbations of BMP/TGF$\beta$ and VEGFNEGFR signalling pathways in non-syndromic sporadic brain arteriovenous malformations (BAVM). J Med Genet. 2018;55(10):675-84.

\section{Publisher's Note}

Springer Nature remains neutral with regard to jurisdictional claims in published maps and institutional affiliations.

Ready to submit your research? Choose BMC and benefit from:

- fast, convenient online submission

- thorough peer review by experienced researchers in your field

- rapid publication on acceptance

- support for research data, including large and complex data types

- gold Open Access which fosters wider collaboration and increased citations

- maximum visibility for your research: over $100 \mathrm{M}$ website views per year

At BMC, research is always in progress.

Learn more biomedcentral.com/submissions 